\title{
The clinical characteristics and prognosis of subgaleal hemorrhage in newborn
}

\author{
Sun Jin Lee, MD', Jin Kyu Kim, MD, PhD ${ }^{1,2,3}$, Sun Jun Kim, MD, PhD ${ }^{1,2,3}$ \\ ${ }^{1}$ Department of Pediatrics, ${ }^{2}$ Research Institute of Clinical Medicine, Chonbuk National University Medical School, Jeonju, ${ }^{3}$ Biomedical Research Institute of Chonbuk \\ National University, Jeonju, Korea
}

Purpose: Subgaleal hemorrhage (SGH) is a rare but potentially fatal condition in newborns; however, few studies have reported on this condition. We aimed to identify the clinical characteristics and prognostic factors of SGH.

Methods: We retrospectively reviewed the medical records of 20 neonates diagnosed with SGH between January 2000 and June 2017. Enrolled neonates were clinically diagnosed when they had tender fluctuant scalp swelling that crossed the suture lines.

Results: Among 20 neonates with SGH, 12 were boys and 7 were girls; median hospitalization duration was $9.7 \pm 6.9$ days. Fourteen neonates (70\%) were born via vacuum-assisted vaginal delivery, and 4 via vacuum-assisted cesarean section. Of the neonates enrolled, half of them initially showed unstable vital signs, including apnea, desaturation, and cyanosis. Ten neonates had acidosis and 3 had asphyxia $(\mathrm{pH}<7.0)$. Intracranial lesions associated with SGH were observed in 15 neonates $(75 \%)$, including subdural hemorrhage (50\%), subarachnoid hemorrhage (15\%), intraventricular hemorrhage (5\%), cerebral infarct $(15 \%)$, skull fracture $(30 \%)$, and cephalohematoma $(20 \%)$. Twelve neonates $(60 \%)$ required transfusion, $5(25 \%)$ had seizures, and $3(15 \%)$ died. Eight neonates (40\%) had hyperbilirubinemia (mean total bilirubin, 13.1 \pm 7.4 ). The mean follow-up period was $8.4 \pm 7.5$ months. At follow-up, 10 neonates (58.8\%) were healthy with normal development, whereas 7 (41.2\%) had neurological deficits.

Conclusion: The morbidity rate was $41.2 \%$ due to severe metabolic acidosis. Anemia, hyperbilirubinemia, low Apgar scores, and subdural hemorrhage did not affect the prognosis. The long-term outcomes of neonates with SGH are generally good. Only arterial blood pH was significantly associated with death.

Key words: Subgaleal hemorrhage, Vacuum extraction delivery

\section{Introduction}

Subgaleal hemorrhage (SGH) is an accumulation of blood in the space between the epicranial aponeurosis and the periosteum, caused by the rupture of the emissary veins. ${ }^{1)}$ The subaponeurotic space is a potential space extending anteriorly to the orbital margins, posteriorly to the nuchal ridge, and laterally to the temporal fascia, and this space may accumulate as much as $260 \mathrm{~mL}$ of blood. ${ }^{2)}$

Meanwhile, cephalhematoma and caput succedaneum can also occur following instrumental delivery. Cephalhematoma is a collection of blood between the skull and its periosteum, and caput succedaneum is an edematous collection of serosanguinous fluid in the subcutaneous layer of the scalp. Cephalhematoma usually involves the parietal or occipital bone, sharply limited by the margins of the bone and does not cross the suture lines. ${ }^{3,4)}$ SGH can be distinguished clinically from cephalhematoma because it extends more widely and crosses the suture lines. ${ }^{5,6)}$ If a diffuse, boggy swelling develops over the scalp of a newborn, not limited
Corresponding author: Sun Jun Kim, MD

Department of Pediatrics, Chonbuk National University Medical School, 567 Baekje-daero, Deokjingu, Jeonju 54896, Korea Tel: +82-63-250-1799

Fax: +82-63-250-1464

E-mail: sunjun@jbnu.ac.kr

https://orcid.org/0000-0002-7673-8728

Received: 24 July, 2018

Revised: 30 August, 2018

Accepted: 3 September, 2018

Copyright @ 2018 by The Korean Pediatric Society

This is an open-access article distributed under the terms of the Creative Commons Attribution NonCommercial License (http://creativecommons.org/ licenses/by-nc/4.0/ which permits unrestricted noncommercial use, distribution, and reproduction in any medium, provided the original work is properly cited. 
by the suture lines, SGH should be considered. ${ }^{4)}$ Early SGH may present a similar clinical pattern as caput succedaneum, but it progresses, in contrast to the rapid resolution of the swelling in caput succedaneum. With progressive SGH, fluctuant mass accompanying pitting edema may shift to the dependent side, resulting in the elevation and displacement of the ear lobe and puffiness of the eyelid. ${ }^{1)}$ Given the severe complications of SGH, such as hypovolemic shock, acute anemia, coagulopathy and death, early detection is needed. ${ }^{1,7)}$ Its incidence has been estimated to be approximately 0.4 in 1,000 spontaneous vaginal deliveries without the use of vacuum or forceps, and 5.9 in 1,000 vacuum-assisted deliveries. $^{8)}$

SGH is a rare and potentially fatal condition in newborns. However, local and international studies on this condition are scarce. Thus, the study aimed to investigate the prognostic factors of SGH by analyzing the clinical characteristics of the disease.

\section{Materials and methods}

In this single-center retrospective study, we evaluated medical records of 20 neonates with SGH who were admitted to the neonatal intensive care unit of Chonbuk National University Children's Hospital between January 2000 and June 2017. The medical records were reviewed to obtain the obstetric and neonatal data (i.e., gestational age, birth weight, and mode of delivery, Apgar score, duration of hospitalization, initial impression, and time of diagnosis).

The diagnosis of SGH was made clinically when tender fluctuant scalp swelling that crosses the suture lines exists. The head circumference was measured at the time of hospitalization and then hourly until there was no increase in the neonates' head circumference. Blood samples were taken from a radial or brachial artery, and capillary blood sampling was performed via heel punctures if arterial blood was difficult to obtain.

Both intracranial and extracranial comorbidities were evaluated using laboratory findings and imaging results during the hospitalization. Hyperbilirubinemia was defined as serum bilirubin level of more than $13 \mathrm{mg} / \mathrm{dL}$. Anemia was defined as serum hemoglobin level $<13 \mathrm{~g} / \mathrm{dL}$. Metabolic acidosis was defined as serum $\mathrm{pH}<7.35$ and serum bicarbonate $\left(\mathrm{HCO}_{3}{ }^{-}\right)<18 \mathrm{mmol} / \mathrm{L}$. Acute kidney injury was defined as serum creatinine $>1.5 \mathrm{mg} / \mathrm{dL}$.

Surviving infants were followed up in the pediatric neurology and neonatal clinics after discharge. The pediatric neurologist completed a neurologic examination of the neonates. Neonates who were suspected to have developmental delay underwent brain magnetic resonance imaging (MRI), electroencephalogram, and language evaluations. The patients were categorized into the following groups according to the results of the examinations: healthy children group included children with normal development and had no diseases, and poor outcome group included children with neurological defi- cits. The mean follow-up period was $8.4 \pm 7.5$ months.

Statistical analysis of the results was performed using IBM SPSS Statistics ver. 22.0 (IBM Co., Armonk, NY, USA). All data were expressed as mean \pm standard deviation. Independent $t$-test was used to assess the factors according to the short-term outcome differences between the healthy children and poor outcome groups.

This study was performed with approval from the Institutional Review Board (IRB) of Chonbuk National University Research Council (CUH 2018-06-027). The requirement for informed consent was exempted by the IRB.

\section{Results}

Three patients (15\%) died during hospitalization. Fourteen neonates $(70 \%)$ were delivered via vacuum extraction delivery, 1 (5\%) via an emergency cesarean section, 1 (5\%) vaginally, and 18 (90\%) via vacuum extraction. Vacuum extraction was required during ce-

Table 1. Baseline clinical characteristics of the study population

\begin{tabular}{|c|c|}
\hline Variable & Value \\
\hline Gestational age (wk) & $38.9 \pm 1.2$ \\
\hline Birth weight (kg) & $3.4 \pm 0.3$ \\
\hline Hospital days (day) & $11.4 \pm 6.1$ \\
\hline \multicolumn{2}{|l|}{ Apgar score } \\
\hline 1 Minute & $6.8 \pm 2.4$ \\
\hline 5 Minutes & $8.3 \pm 2.1$ \\
\hline Sex, male:female & $12: 8(60: 40)$ \\
\hline Transferred from other hospitals & $18(90)$ \\
\hline \multicolumn{2}{|l|}{ Mode of delivery } \\
\hline Vaginal delivery & $1(5)$ \\
\hline Vacuum extractor delivery & $14(70)$ \\
\hline Emergency cesarean section & $1(5)$ \\
\hline Vacuum during cesarean section & $4(20)$ \\
\hline \multicolumn{2}{|l|}{ Complete blood count } \\
\hline Initial hemoglobin (g/dL) & $13.9 \pm 3.8$ \\
\hline Initial hematocrit (\%) & $41.7 \pm 11.2$ \\
\hline Initial platelet count $\left(\times 10^{3} / \mu \mathrm{L}\right)$ & $230.7 \pm 70.9$ \\
\hline \multicolumn{2}{|l|}{ Arterial blood gas analysis } \\
\hline Initial pH & $7.26 \pm 0.22$ \\
\hline Initial $\mathrm{HCO}_{3}(\mathrm{mmol} / \mathrm{L})$ & $15.8 \pm 4.9$ \\
\hline Initial $\mathrm{pCO}_{2}(\mathrm{mmHg})$ & $37.8 \pm 31.6$ \\
\hline Initial $\mathrm{pO}_{2}(\mathrm{mmHg})$ & $104.3 \pm 74.9$ \\
\hline Initial lactate (mmol/L) & $8.8 \pm 4.9$ \\
\hline \multicolumn{2}{|l|}{ Chemistry } \\
\hline Maximum total bilirubin (mg/dL) & $13.1 \pm 7.4$ \\
\hline High sensitive CRP (mg/L) & $1.2 \pm 2.0$ \\
\hline
\end{tabular}


sarean section in 4 cases (20\%). The mean gestational age at birth was 38.9 \pm 1.2 weeks. The mean Apgar scores were $6.8 \pm 2.4$ at $1 \mathrm{~min}$ ute and $8.3 \pm 2.1$ at 5 minutes (Table 1).

Eighteen of the 20 neonates (90\%) were transferred from another hospital. The initial presenting symptoms at admission varied, with scalp swelling (20\%) being the most common. Half of the patients presented apnea, desaturation, tachypnea, moaning, and cyanosis as the initial symptom (Fig. 1). Initial mean hemoglobin level and mean arterial blood gas $\mathrm{pH}$ were $13.9 \mathrm{~g} / \mathrm{dL}$ and $7.26 \pm 0.22$, respectively (Table 1).

Comorbid pathology identified by neuroimaging is outlined in Table 2. Of the 20 neonates, 12 (60\%) underwent brain sonography,

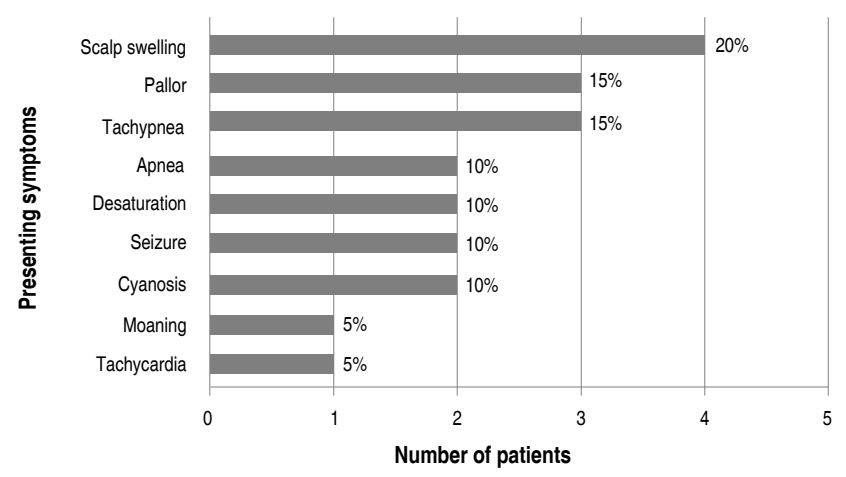

Fig. 1. Initial presenting symptoms in neonates.

Table 2. Comorbidities in the neonates

\begin{tabular}{lc}
\hline Variable & No. $(\%)$ \\
\hline Imaging modality & \\
Brain sonography & $12(60)$ \\
Brain computed tomography & $5(25)$ \\
Brain magnetic resonance imaging & $8(40)$ \\
Intracranial comorbidities & \\
Subdural hematoma & $10(50)$ \\
Intraventricular hemorrhage & $2(5)$ \\
Cerebral infarction & $3(15)$ \\
Skull fracture & $5(25)$ \\
Anemia & $12(60)$ \\
Hyperbilirubinemia & $8(40)$ \\
Metabolic acidosis & \\
$7.25 \leq$ pH $<7.35$ & $4(20)$ \\
$7.10 \leq$ pH<7.25 & $2(10)$ \\
$7.0 \leq$ pH $<7.10$ & $1(5)$ \\
pH<7.0 & $3(15)$ \\
Seizure & $5(25)$ \\
Disseminated intravascular coagulation & $3(15)$ \\
Acute kidney injury & $2(10)$ \\
Brachial plexus injury & $1(5)$ \\
Hip dislocation & $1(5)$ \\
\hline
\end{tabular}

5 (25\%) had brain computed tomography (CT), and 8 (40\%) had brain MRI. Ten neonates (50\%) had subdural hemorrhage (SDH), 2 (5\%) had intraventricular hemorrhage, 3 (15\%) showed cerebral infarction, and 5 (25\%) had skull fractures (Table 2).

The most common extracranial comorbidities were anemia (60\%). Ten neonates (50\%) had metabolic acidosis and 3 (15\%) had severe asphyxia ( $\mathrm{pH}<7.0)$. Five neonates (25\%) had seizures, 3 (15\%) had disseminated intravascular coagulation, 2 (10\%) had acute kidney injury, 1 (5\%) had brachial plexus injury, and 1 (5\%) had hip dislocation (Table 2).

The mean follow-up period was $8.4 \pm 7.5$ months. In a clinical follow-up, 2 subgroups were analyzed according to the shortterm outcome. Of the 17 neonates who survived, 10 (58.8\%) were categorized in the healthy children group, whereas 7 (41.2\%) were categorized in the poor outcome group. In the poor outcome group, 1 neonate (5.9\%) was diagnosed with spastic cerebral palsy (CP), 2 (11.8\%) with spastic CP and epilepsy, 2 (11.8\%) with hypotonic $\mathrm{CP}$ accompanied by global developmental delay. The patient with brachial plexus injury had an accompanying monoparesis of the left upper limb (Table 3). When comparing the 2 subgroups, the mean gestational age was significantly increased in the healthy children group $(P=0.035)$. Hospitalization duration was $8.2 \pm 2.3$ days in the healthy children group, whereas it was $15.9 \pm 7.1$ days in the poor outcome group, indicating that the poor outcome group had a significantly longer hospitalization duration $(P=0.029)$. There were no significant differences in the Apgar score, duration of mechanical ventilation use, and presence of SDH (Table 4).

In terms of the time of diagnosis, 10 neonates (58.8\%) were diagnosed early at the time of admission, whereas 7 (41.2\%) were diagnosed the following day after their admission (Fig. 2). Of the 10 neonates diagnosed on the day of admission, 8 (47.1\%) were in the healthy children group and $2(11.8 \%)$ were in the poor outcome group. Of the 7 children who were diagnosed a day after the admission, 2 (11.8\%) were in the healthy children group and 5 (29.4\%) were in the poor outcome group (Fig. 2).

Table 3. Short-term outcome of surviving children

\begin{tabular}{lc}
\hline Variable & Value \\
\hline Follow-up periods (mo) & $8.4 \pm 7.5$ \\
Healthy with normal development & $10(58.8)$ \\
Spastic CP & $1(5.9)$ \\
Spastic CP with epilepsy & $2(11.8)$ \\
Hypotonic CP with GDD & $2(11.8)$ \\
Language developmental delay & $1(5.9)$ \\
Monoparesis & $1(5.9)$ \\
\hline Values are presented as mean \pm standard deviation or number (\%). &
\end{tabular}


Table 4. Comparisons of clinical factors in 2 subgroups according to short-term outcome

\begin{tabular}{lcrc}
\hline Variable & $\begin{array}{c}\text { Healthy children } \\
(\mathrm{n}=10)\end{array}$ & $\begin{array}{c}\text { Poor outcome } \\
(\mathrm{n}=7)\end{array}$ & $P$ value \\
\hline Gestational age $(\mathrm{wk})$ & $39.5 \pm 0.8$ & $38.2 \pm 1.4$ & 0.035 \\
Birth weight $(\mathrm{kg})$ & $3.5 \pm 0.3$ & $3.2 \pm 0.4$ & 0.074 \\
Hospitalization period (day) & $8.2 \pm 2.3$ & $15.9 \pm 7.1$ & 0.029 \\
Apgar score & & & \\
$\quad 1$ Minute & $7.6 \pm 2.0$ & $6.6 \pm 1.9$ & 0.298 \\
$\quad 5$ Minutes & $9.0 \pm 1.4$ & $8.3 \pm 1.4$ & 0.317 \\
Hemoglobin $(\mathrm{g} / \mathrm{dL})$ & $14.1 \pm 4.8$ & $13.7 \pm 3.2$ & 0.843 \\
Platelet $\left(\times 10^{3} / \mathrm{\mu L}\right)$ & $242.7 \pm 59.9$ & $245.1 \pm 71.6$ & 0.918 \\
ABGA pH & $7.37 \pm 0.1$ & $7.27 \pm 0.2$ & 0.277 \\
Duration of mechanical ventilation & $0.3 \pm 0.9$ & $2.9 \pm 3.7$ & 0.117 \\
$\quad$ use (day) & & & \\
Presence of SDH & $5(50.0)$ & $5(71.4)$ & 0.622 \\
Diagnosed on admission day & $8(80.0)$ & $5(71.4)$ & 0.622 \\
\hline
\end{tabular}

Values are presented as mean \pm standard deviation or number (\%). ABGA, arterial blood gas analysis; SDH, subdural hemorrhage.

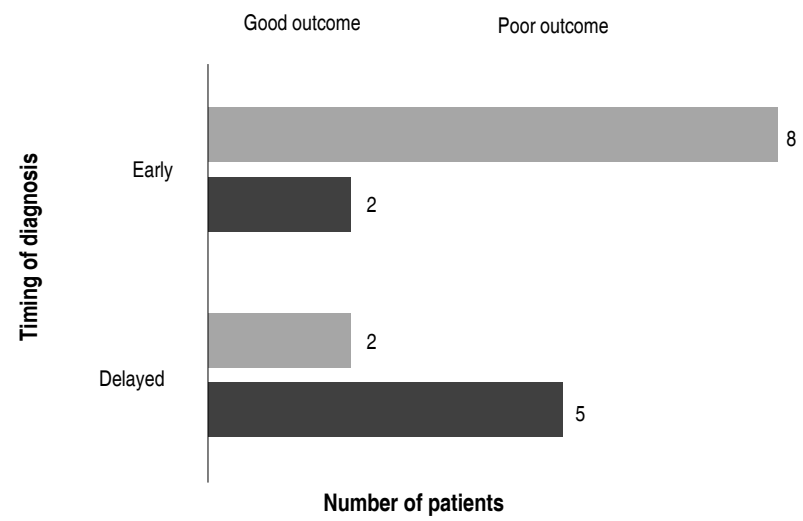

Fig. 2. Classification of outcomes according to diagnostic timing in the surviving children.

\section{Discussion}

In this retrospective study, we demonstrated that $41.2 \%$ of neonates with SGH had comorbidities, including anemia, subdural hematoma, hyperbilirubinemia, and metabolic acidosis. The children who had seizures or disseminated intravascular coagulation showed poor outcomes in the short-term follow-up. The mortality rate was 15\%. The mortality cases had the following common characteristics: delivered via vacuum-assisted vaginal delivery and diagnosed with severe SGH. They had severe metabolic acidosis $(\mathrm{pH}<7.0)$ in the initial arterial blood gas analysis. All neonates were transferred from other hospitals on the day of birth, with one neonate dying upon arrival and 2 neonates dying within 48 hours after birth.

Swanson et al. ${ }^{9)}$ reported 21 neonates with SGH with three mortality cases, similar to our case series. However, it is difficult to predict the neurological outcome because only half of the surviving children in that study were followed up. In this study, all surviving children were followed up, with the majority of children having a normal development and without neurological deficits. The neurological sequelae include seizures, neurodevelopmental delay, and CP. Similarly, other previous studies have shown that the long-term outcomes of neonates with SGH are generally good., ${ }^{7-11)}$

We also examined the association between the short-term outcomes and the timing of SGH diagnosis. Children who were diagnosed with SGH at the day of admission have shown to have better prognosis than those who were diagnosed the following the day after their admission. In other words, although the symptoms were mild at first, the patients with suspected SGH who underwent repeated examinations and tests early had a good prognosis, whereas those with symptomatic SGH had poor outcomes. The good prognosis is considered to be due to timely and proper treatment following the early diagnosis, which is consistent with the results of other studies. ${ }^{2,7,12)}$ As already known, most cases of SGH are related to the use of vacuum extraction. In this study, all neonates were delivered using a vacuum extractor, except for 2 neonates who were delivered via spontaneous vaginal delivery and emergency cesarean section. In neonates with SGH who were delivered via a nontraumatic delivery, the possibility of coagulopathy should be considered. ${ }^{13)}$

The factors leading to the poor outcomes of SGH were reported in several studies. Chang et al. ${ }^{14)}$ demonstrated that hypotension, seizure, anemia, coagulopathy, metabolic acidosis, and renal impairment are predictive factor of poor outcomes. $\mathrm{Ng}$ et al. ${ }^{15)}$ reported that the most important factors related to the mortality of the neonates with severe SGH were birth asphyxia with arterial cord blood $\mathrm{pH}<7.20$ and Apgar score of less than 3 at 1 minute after birth. This present study showed that the longer gestational age and the shorter hospitalization duration were related to good prognosis when comparing the two subgroups according to the short-term outcome. In contrast, the duration of mechanical ventilation use, presence of SDH, anemia, hyperbilirubinemia, and renal impairment showed no correlation with the outcome. In our 3 fetal death cases, only arterial blood $\mathrm{pH}$ was significantly associated with deaths. Given that the Apgar scores of the 2 mortality cases were not recorded, the association between Apgar score and death was not evaluated.

In this study, half of the cases were accompanied by SDH, but the presence of SDH was not significantly related to the neurologic outcome. Similarly, Kilani and Wetmore ${ }^{12)}$ reported that severe hypovolemia and coagulopathy were the most commonly associated clinical factors with mortality, but intracranial hemorrhage had no relationship with death in neonates with SGH.

This study has some limitations. A standardized assessment tool to assess the children's neurodevelopment status was absent; hence, it is possible that a mere clinical neurological examination is not reliable enough to detect any minor neurologic deficit. The follow-up period varied from each patient and was relatively short 
duration. It also cannot exclude that the number of patients enrolled in this study is too small to assess the factors affecting the outcome of the SGH. Thus, further studies enrolling larger patient numbers with long-term follow-up is warranted. Moreover, not all neonates underwent the imaging examinations to confirm the diagnosis; only 85\% underwent imaging, 35.3\% whom only had ultrasonography. Although the imaging study may be helpful in assessing the severity of the disease, it is not necessary for diagnosis. Similar to other studies, the diagnosis was based on clinical features, and ultrasonography or CT was not used to confirm the diagnosis. ${ }^{10,15-18)}$

In conclusion, SGH initially presents with various symptoms, but if overlooked, it progresses gradually, resulting in serious neurological sequelae or death. Early diagnosis and immediate management are important to improve the outcomes among neonates with SGH. Close monitoring, including hourly recordings of vital signs and head circumference measurements, is required for all infants with unstable vital signs, as well as those who are delivered via vacuum extractions.

\section{Conflicts of interest}

No potential conflict of interest relevant to this article was reported.

\section{References}

1. The Royal Australian and New Zealand College of Obstetricians and Gynaecologists. Prevention, detection, and management of subgaleal haemorrhage in the newborn C-Obs 28. East Melbourne (Australia): The Royal Australian and New Zealand College of Obstetricians and Gynaecologists, 2009.

2. Davis DJ. Neonatal subgaleal hemorrhage: diagnosis and manage- ment. CMAJ 2001;164:1452-3.

3. Nicholson L. Caput succedaneum and cephalohematoma: the cs that leave bumps on the head. Neonatal Netw 2007;26:277-81.

4. Reid J. Neonatal subgaleal hemorrhage. Neonatal Netw 2007;26:21927.

5. Kokolakis M, Koutelekos I. Birth related traumatic brain injury - nursing interventions. Perioper Nursing 2015;4:121-37.

6. Rabelo NN, Matushita H, Cardeal DD. Traumatic brain lesions in newborns. Arq Neuropsiquiatr 2017;75:180-8.

7. Colditz MJ, Lai MM, Cartwright DW, Colditz PB. Subgaleal haemorrhage in the newborn: A call for early diagnosis and aggressive management. J Paediatr Child Health 2015;51:140-6.

8. Plauché WC. Subgaleal hematoma. A complication of instrumental delivery. JAMA 1980;244:1597-8.

9. Swanson AE, Veldman A, Wallace EM, Malhotra A. Subgaleal hemorrhage: risk factors and outcomes. Acta Obstet Gynecol Scand 2012; 91:260-3.

10. Chadwick LM, Pemberton PJ, Kurinczuk JJ. Neonatal subgaleal haematoma: associated risk factors, complications and outcome. J Paediatr Child Health 1996;32:228-32.

11. Nasseri F, Badiei Z. Neonatal subgaleal hemorrhage "A fetal complication of vacuum extraction delivery”. Iran J Ped 2007;17:297-301.

12. Kilani RA, Wetmore J. Neonatal subgaleal hematoma: presentation and outcome--radiological findings and factors associated with mortality. Am J Perinatol 2006;23:41-8.

13. Wetzel EA, Kingma PS. Subgaleal hemorrhage in a neonate with factor X deficiency following a non-traumatic cesarean section. J Perinatol 2012;32:304-5.

14. Chang HY, Peng CC, Kao HA, Hsu CH, Hung HY, Chang JH. Neonatal subgalealhemorrhage: clinical presentation, treatment, and predictors of poor prognosis. Pediatr Int 2007;49:903-7.

15. Ng PC, Siu YK, Lewindon PJ. Subaponeurotic haemorrhage in the 1990s: a 3-year surveillance. Acta Paediatr 1995;84:1065-9.

16. Boo NY, Foong KW, Mahdy ZA, Yong SC, Jaafar R. Risk factors associated with subaponeurotic haemorrhage in full-term infants exposed to vacuum extraction. BJOG 2005;112:1516-21.

17. Boo NY. Subaponeurotic haemorrhage in Malaysian neonates. Singapore Med J 1990;31:207-10.

18. Gebremariam A. Subgaleal haemorrhage: risk factors and neurological and developmental outcome in survivors. Ann Trop Paediatr 1999;19:45-50. 